\title{
MANY SYMMETRICALLY INDIVISIBLE STRUCTURES
}

\author{
NADAV MEIR
}

\begin{abstract}
A structure $\mathcal{M}$ in a first-order language $\mathcal{L}$ is indivisible if for every colouring of its universe $M$ in two colours, there is a monochromatic $\mathcal{M}^{\prime} \subseteq \mathcal{M}$ such that $\mathcal{M}^{\prime} \cong \mathcal{M}$. Additionally, we say that $\mathcal{M}$ is symmetrically indivisible if $\mathcal{M}^{\prime}$ can be chosen to be symmetrically embedded in $\mathcal{M}$ (that is, every automorphism of $\mathcal{M}^{\prime}$ can be extended to an automorphism of $\mathcal{M}$ ). In the following paper we give a general method for constructing new symmetrically indivisible structures out of existing ones. Using this method, we construct $2^{\aleph_{0}}$ many non-isomorphic symmetrically indivisible countable structures in given (elementary) classes and answer negatively the following question from HKO11: Let $\mathcal{M}$ be a symmetrically indivisible structure in a language $\mathcal{L}$. Let $\mathcal{L}_{0} \subseteq \mathcal{L}$. Is $\mathcal{M}\left\lceil\mathcal{L}_{0}\right.$ symmetrically indivisible?
\end{abstract}

\section{INTRODUCTION}

The notion of indivisibility of relational first-order structures and metric spaces is well studied in Ramsey theory ( KR86, EZS91, [EZS93] are just a few examples of the extensive study in this area). Recall that a structure $\mathcal{M}$ in a relational first-order language is indivisible, if for every colouring of its universe $M$ in two colours, there is a monochromatic substructure $\mathcal{M}^{\prime} \subseteq \mathcal{M}$ such that $\mathcal{M}^{\prime} \cong \mathcal{M}$. Rado's random graph, the ordered set of natural numbers and the ordered set of rational numbers are just a few of the many examples. Weakenings of this notion have also been studied (see [Sau14]). A known extensively studied strengthening of this notion is the pigeonhole property. A first-order relational structure $X$ admits the pigeonhole property if whenever $X$ is the union of two disjoint substructures $Y$ and $Z$, at least one of $Y$ and $Z$ is isomorphic to $X$. Examples of such structures include the random graph and the random $n$-hypergraph, though in general such structures are very rare. (See Cam10] for further reading.) For an extensive review on the subject - see appendix A in Fra00.

In GK11, a notion of symmetrized Ramsey theory was introduced, and in HKO11 a new strengthening of the notion of indivisibility was investigated: We say that a substructure $\mathcal{N} \subseteq \mathcal{M}$ is symmetrically embedded in $\mathcal{M}$ if every automorphism of $\mathcal{N}$ extends to an automorphism of $\mathcal{M}$. We say that $\mathcal{M}$ is symmetrically indivisible if for every colouring of $M$ in two colours, there is a monochromatic $\mathcal{M}^{\prime} \subseteq \mathcal{M}$ such that $\mathcal{M}^{\prime}$ is isomorphic to $\mathcal{M}$ and $\mathcal{M}^{\prime}$ is symmetrically embedded in $\mathcal{M}$.

In [HKO11, several examples of symmetrically indivisible structures were introduced. Examples include the random graph (GK11]), the ordered rational numbers, the ordered natural numbers, the universal $n$-hypergraph.

2010 Mathematics Subject Classification. 05C15, 05C55, 03C07, $20 \mathrm{~B} 27$.

Key words and phrases. Indivisibility, Symmetric indivisibility, Colouring, Automorphism. 
In Section 2 we will present a method of constructing new symmetrically indivisible structures out of existing ones and using this method, construct $2^{\aleph_{0}}$ many non-isomorphic symmetrically indivisible countable linear orders and $2^{\aleph_{0}}$ many non-isomorphic symmetrically indivisible countable graphs. We give a sufficient conditions for a class of $\mathcal{L}$-structures to have $2^{\aleph_{0}}$ many symmetrically indivisible structures. We note that these conditions are met by the class of $n$-hypergraphs, graphs edge-coloured in $k \leq \omega$ colours, and trivially by the class of partial orders as a super-class of linear orders and with an aim of finding a general claim.

In Section 3 we make further use of this method to construct an example that answers negatively a question asked in [HKO11]: Let $\mathcal{M}$ be a symmetrically indivisible structure in a language $\mathcal{L}$. Let $\mathcal{L}_{0} \subseteq \mathcal{L}$. Is $\mathcal{M} \uparrow \mathcal{L}_{0}$ symmetrically indivisible? It is clear that if $\mathcal{M}$ is indivisible then $\mathcal{M} \uparrow \mathcal{L}_{0}$ is indivisible, but for a symmetrically embedded $\mathcal{M}_{0} \subseteq \mathcal{M}, \mathcal{M}_{0}\left\lceil\mathcal{L}_{0}\right.$ is not necessarily symmetrically embedded in $\mathcal{M}$, thus this question does not seem to have an immediate answer.

\section{MANY EXAMPLES FOR SYMMETRICALLY INDIVISIBLE STRUCTURES}

In this section we will show how to construct many symmetrically indivisible structures based on existing ones.

Definition 2.1. For two first-order structures $\mathcal{M}, \mathcal{N}$ an embedding $e: \mathcal{N} \rightarrow \mathcal{M}$ is called symmetric if every automorphism of $e[\mathcal{N}]$ extends to an automorphism of $\mathcal{M}$. So a substructure $\mathcal{N} \subset \mathcal{M}$ is symmetrically embedded if the inclusion map $\iota$ is symmetric.

Notation 2.2. Let $\mathcal{L}$ be a first-order language and let $\mathcal{M}, \mathcal{N}$ be $\mathcal{L}$-structures. We write $\mathcal{M} \lesssim \mathcal{N}$ if there is an embedding $e: \mathcal{M} \hookrightarrow \mathcal{N}$. In the same fashion we write $\mathcal{M} \lesssim s \mathcal{N}$ if there is a symmetric embedding $e: \mathcal{M} \hookrightarrow \mathcal{N}$.

Similarly, we write $\mathcal{M} \subseteq \mathcal{N}$ if $\mathcal{M}$ is a substructure of $\mathcal{N}$ and $\mathcal{M} \subseteq{ }_{s} \mathcal{N}$ if there is a symmetric embedding $e: \mathcal{M} \hookrightarrow \mathcal{N}$.

Finally, $\mathcal{M} \sim \mathcal{N}$ means that both $\mathcal{M} \lesssim \mathcal{N}$ and $\mathcal{N} \lesssim \mathcal{M}$ hold, and $\mathcal{M} \sim_{s} \mathcal{N}$ means that both $\mathcal{M} \lesssim_{s} \mathcal{N}$ and $\mathcal{N} \lesssim_{s} \mathcal{M}$ hold

\section{proposition 2.3.}

(1) If $\mathcal{M}$ and $\mathcal{N}$ are $\mathcal{L}$-structures such that $\mathcal{M} \sim \mathcal{N}$ then $\mathcal{M}$ is indivisible iff $\mathcal{N}$ is indivisible.

(2) If $\mathcal{M}$ and $\mathcal{N}$ are $\mathcal{L}$-structures such that $\mathcal{M} \sim_{s} \mathcal{N}$ then $\mathcal{M}$ is symmetrically indivisible iff $\mathcal{N}$ is symmetrically indivisible.

Proof.

(2) Because $\sim_{s}$ is an equivalence relation, it is enough to show one direction: suppose $\mathcal{M}$ is symmetrically indivisible, and let $c: \mathcal{N} \rightarrow\{$ red, blue $\}$ be a colouring of $\mathcal{N}$. Since $\mathcal{M} \lesssim s \mathcal{N}$, let $\mathcal{M}_{0} \subseteq_{s} \mathcal{N}$ be such that $\mathcal{M}_{0} \cong \mathcal{M}$, so $c \uparrow \mathcal{M}_{0}$ is a colouring of $\mathcal{M}_{0}$, and since $\mathcal{M}$ is symmetrically indivisible, so is $\mathcal{M}_{0}$ and there is a monochromatic $\mathcal{M}_{0}^{\prime} \subseteq{ }_{s} \mathcal{M}_{0}$ such that $\mathcal{M}_{0}^{\prime}$ is isomorphic to $\mathcal{M}_{0} \cong \mathcal{M}$. Now, since $\mathcal{N} \lesssim s \mathcal{M} \cong \mathcal{M}_{0}^{\prime}$, there is $\mathcal{N}_{0} \subseteq s \mathcal{M}_{0}^{\prime}$ such that $\mathcal{N}_{0}$ is isomorphic to $\mathcal{N}$ and since $\mathcal{M}_{0}^{\prime}$ is monochromatic, so is $\mathcal{N}_{0}$. Now, since $\mathcal{N}_{0} \subseteq_{s} \mathcal{M}_{0}^{\prime} \subseteq_{s} \mathcal{M}_{0} \subseteq_{s} \mathcal{N}$, by transitivity $\mathcal{N}_{0} \subseteq_{s} \mathcal{N}$.

(1) Repeat the same argument, omitting "symmetric". 
Now we are ready to construct $2^{\aleph_{0}}$ examples of symmetrically indivisible graphs and $2^{\aleph_{0}}$ examples of symmetrically indivisible linear orders, both based on known symmetrically indivisible structures, and the equivalence relation $\sim_{s}$ :

Recall:

Fact 2.4. the random graph and $(\mathbb{Q},<)$ are symmetrically indivisible. (GK11, [HKO11])

In Hen71 it was shown that:

Fact 2.5. Let $\Gamma$ be the random graph. For every countable graph $G, G \lesssim_{s} \Gamma$.

Corollary 2.6. Every countable graph which symmetrically embeds $\Gamma$ is symmetrically indivisible.

Proof. Let $G$ be a countable graph which symmetrically embeds $\Gamma$. Then by definition $G \sim_{s} \Gamma$ thus by Fact 2.4 combined with Proposition 2.3, $G$ is symmetrically indivisible.

For $(\mathbb{Q},<)$ we have a result similar to Fact 2.5 .

proposition 2.7. For every countable linear order $A, A \lesssim_{s} \mathbb{Q}$.

Proof. Let $A[\mathbb{Q}]$ be the lexicographic order on $A \times \mathbb{Q}$. This is a countable dense linear order without end-points $(\mathrm{DLO})$. By $\aleph_{0}$-categoricity of DLO, it is isomorphic to $\langle\mathbb{Q},<\rangle$.

For a fixed $q \in \mathbb{Q}$, the induced substructure on $A \times\{q\}$ is isomorphic to $A$ and the fact that it is symmetrically embedded can be easily verified and is actually a special case of Lemma 2.8 of [HKO11.

From this we have, exactly like Corollary 2.6 for graphs :

Corollary 2.8. Every countable linear order which symmetrically embeds $(\mathbb{Q},<)$ is symmetrically indivisible.

Definition 2.9. Let $G, H$ be graphs and for convenience assume $|G| \cap|H|=\emptyset$. We define $G+{ }^{\mathcal{G}} H$ to be the graph whose universe is $|G| \cup|H|$ and $E^{\left(G+{ }^{\mathcal{G}} H\right)}:=E^{G} \cup E^{H}$.

Namely, $G+{ }^{\mathcal{G}} H$ is just the "disjoint union of graphs" as known in graph theory and denoted by $G \cup H$.

Remark 2.10. Let $G, H, K$ be graphs.

(1) $G, H \lesssim_{s} G+{ }^{\mathcal{G}} H$

(2) $G+{ }^{\mathcal{G}} H=H+{ }^{\mathcal{G}} G$

(3) $\left(G+{ }^{\mathcal{G}} H\right)+{ }^{\mathcal{G}} K=G+{ }^{\mathcal{G}}\left(H+{ }^{\mathcal{G}} G\right)$

(4) If $C \subseteq G$ is a union of connected components, then $G=(G \backslash C)+{ }^{\mathcal{G}} C$

proposition 2.11. If $G, H$ are graphs such that $\Gamma+{ }^{\mathcal{G}} G \cong \Gamma+{ }^{\mathcal{G}} H$, then $G \cong H$.

Proof. Let $\phi: \Gamma+{ }^{\mathcal{G}} G \rightarrow \Gamma+{ }^{\mathcal{G}} H$ be an isomorphism. Since $\phi$ maps connected components onto connected components and $\Gamma$ is connected, either $\phi[\Gamma]=\Gamma$ or $\phi[\Gamma] \subseteq H$. In the first case $\phi[G]=H$ and $\phi\lceil G: G \rightarrow H$ is an isomorphism. In the second case, by Remark 2.10

$$
H+{ }^{\mathcal{G}} \Gamma=(H \backslash \phi[\Gamma])+{ }^{\mathcal{G}} \phi[\Gamma]+{ }^{\mathcal{G}} \Gamma
$$

thus $\phi\left\lceil G: G \rightarrow(H \backslash \phi[\Gamma])+{ }^{\mathcal{G}} \Gamma\right.$ is an isomorphism, but $(H \backslash \phi[\Gamma])+{ }^{\mathcal{G}} \Gamma \cong H$. 
Definition 2.12. Let $A$ and $B$ be linear orders, and for convenience assume $|A| \cap$ $|B|=\emptyset$. We define $A+{ }^{l o} B$ the linear order whose universe is $|A| \cup|B|$ and

$$
<{ }^{A+{ }^{l o} B}:=<^{A} \cup<^{B} \cup\{(a, b) \mid a \in A \text { and } b \in B\} .
$$

Namely, $A+{ }^{l o} B$ is the "concatenation" of $A$ and $B$ - just putting $B$ right after $A$.

proposition 2.13. If $X$ is a linear order such that $|X|=\{x, y\},<^{X}=(x, y)$, and $A, B$ are linear orders such that $\left(\mathbb{Q}+{ }^{l o} X+{ }^{l o} A\right) \cong\left(\mathbb{Q}+{ }^{l o} X+{ }^{l o} B\right)$, then $A \cong B$.

Proof. Let $\phi:\left(\mathbb{Q}+{ }^{l o} X+{ }^{l o} A\right) \rightarrow\left(\mathbb{Q}+{ }^{l o} X+{ }^{l o} B\right)$ be an isomorphism. Now $x$ is the minimal element with an immediate successor and $y$ is the immediate succesor of $x$ in both losets, thus $\phi(x)=x$, and $\phi(y)=y$. Thus

$$
\phi[A]=\phi\left\{z \in \mathbb{Q}+{ }^{l o} X+{ }^{l o} A \mid y<z\right\}=\left\{z \in \mathbb{Q}+{ }^{l o} X+{ }^{l o} B \mid y<z\right\}=B
$$

Similarly to Remark 2.10

Remark 2.14. If $A, B$ are linear orders, then $A, B \lesssim_{s}\left(A+{ }^{l o} B\right)$

\section{Corollary 2.15.}

(1) There is a 1-1 map between isomorphism classes of countable graphs and isomorphism classes of countable symmetrically indivisible graphs.

(2) There is a 1-1 map between isomorphism classes of countable losets and isomorphism classes of countable symmetrically indivisible losets.

Proof. Consider the maps:

(1) $G \mapsto \Gamma+{ }^{\mathcal{G}} G$

(2) $A \mapsto \mathbb{Q}+{ }^{l o}\{x\}+{ }^{l o}\{y\}+{ }^{l o} A$

By Propositions 2.11 and 2.13 these are 1-1. By Remark 2.10 and Corollary 2.6, all the graphs of the form $\Gamma+{ }^{G} G$ are symmetrically indivisible. By Remark 2.14 and Corollary 2.8 all the losets of the form $\mathbb{Q}+{ }^{l o}\{x\}+{ }^{l o}\{y\}+{ }^{l o} A$ are symmetrically indivisible.

We conclude this section with an attempt to generalize both constructions. As mentioned in the introduction, classic examples of symmetrically indivisible structure, in addition to the random graph and the ordered rational numbers, include the universal $n$-hypergraph and the universal edge-coloured graph in $k \leq \omega$ many colours defined below:

Definition 2.16. For $k \leq \omega$, an edge-coloured graph in $k$ many colours $G$ is a graph whose edges are coloured in $k$ many colours - i.e. it is a structure in the language $\mathcal{L}_{k}:=\left\{R_{i}\right\}_{i \in k}$ such that $\left\{G\left\lceil R_{i}\right\}_{i \in k}\right.$ are edge-disjoint graphs.

For a fixed $k$, the class of finite edge-coloured graphs in $k$ many colours is a Fraïssé class. We denote its Fraïssé limit by $\Gamma_{k}$.

In an attempt to generalize Corollary 2.15, we haven't had much success in giving an interesting generalization other than the trivial one, which goes as follows:

proposition 2.17. Assume $\mathcal{C}$ is a class of countable structures in a fixed language $\mathcal{L}$, along with a symmetrically indivisible structure $\mathcal{M} \in \mathcal{C}$ such that for every $C \in \mathcal{C}$, $C \lesssim_{s} \mathcal{M}$ and a binary operation on $\mathcal{C},+{ }^{\mathcal{C}}$ satisfying $\mathcal{M}+{ }^{\mathcal{C}} C_{1} \cong \mathcal{M}+{ }^{\mathcal{C}} C_{2} \Longrightarrow$ $C_{1} \cong C_{2}$ and $A, B \lesssim s A+{ }^{\mathcal{C}} B$. Then there is a 1-1 map between structures of $\mathcal{C}$ up to isomorphism and symmetrically indivisible structures of $\mathcal{C}$ up to isomorphism. 
Regarding $n$-hypergraphs and edge-coloured graphs: A similar construction to that of Theorem 3.1 in Hen71 can give us a result similar to Fact 2.5 and Proposition 2.7

(1) The universal $n$-hypergraph symmetrically embeds every $n$-hypergraph.

(2) $\Gamma_{k}$ symmetrically embeds every edge-coloured graph in $k$ colours.

For $n$-hypergraphs and edge-coloured graphs, + will be just the disjoint union, similar to $+{ }^{\mathcal{G}}$. Proposition 2.17 gives us a 1-1 map between $n$-hypergraphs up to isomorphism and symmetrically indivisible hypergraphs up to isomorphism and the same for edge-coloured graphs in $k \leq \omega$ colours.

\section{A Symmetrically Indivisible Structure with a Reduct that is NOT SYMmetrically Indivisible}

Recall that in [HKO11] the following question was asked:

Question 3.1. Let $\mathcal{M}$ be a symmetrically indivisible structure in a language $\mathcal{L}$. Let $\mathcal{L}_{0} \subseteq \mathcal{L}$. Is $\mathcal{M} \uparrow \mathcal{L}_{0}$ symmetrically indivisible?

In this section, we will construct an example answering this question negatively.

First we construct an indivisible structure which is not symmetrically indivisible. The existence of such a structure is a necessary condition for the existence of an example for Question 3.1, since if $\mathcal{M}$ is indivisible (in particular if it is symmetrically indivisible) then $\mathcal{M} \uparrow \mathcal{L}_{0}$ is also indivisible.

3.1. $\Gamma^{*}-$ an example of an indivisible structure which is not symmetrically indivisible.

Throughout this subsection $\Gamma$ will denote the random graph. The indivisibility of the random graph is a well known fact that dates back to its definition in Rad64. An easy proof is given in Hen71.

Definition 3.2. We define the graph $\Gamma^{*}$ as follows:

Let $\left\{K_{n}\right\}_{n<\omega}$ be disjoint sets, satisfying $\left|K_{n}\right|=n$ and let $\left\{g_{n}\right\}_{n<\omega}$ be an enumeration of $\Gamma$. The universe of $\Gamma^{*}$ is defined to be $\left|\Gamma^{*}\right|=|\Gamma| \cup \bigcup_{n<\omega} K_{n}$ and the edges are defined as follows:

$$
E^{\Gamma^{*}}=E^{\Gamma} \cup \bigcup_{n<\omega}\left\{(a, b) \mid a, b \in K_{n} \cup\left\{g_{n}\right\}, a \neq b\right\}
$$

In words, if $\left\{g_{n}\right\}_{n \in \omega}$ enumerates the vertices of $\Gamma$, for each $n \in \omega$ we add a clique $K_{n}$ and connect it to $g_{n}$.

Lemma 3.3. $\Gamma^{*}$ is not rigid

Proof. for every $n \geq 2$ and for every two distinct $a, b \in K_{n}$, there is an automorphism of $\Gamma^{*}$ swapping $a$ with $b$ and fixing all other vertices.

Lemma 3.4. If $\sigma: \Gamma^{*} \rightarrow \Gamma^{*}$ is an automorphism of $\Gamma^{*}$ then $\sigma \uparrow \Gamma=I d_{\Gamma}$

Proof. For each $n \in \omega, K_{n}$ is the set of vertices in $\Gamma^{*}$ of degree precisely $n$, and $g_{n}$ is the unique vertex of infinite degree connected to all vertices in $K_{n}$. Thus $\sigma\left(g_{n}\right)=g_{n}$.

proposition 3.5. $\Gamma^{*}$ is indivisible but not symmetrically indivisible. 
Proof. First, $\Gamma^{*}$ is indivisible, since clearly $\Gamma \lesssim \Gamma^{*}$ and by Fact 2.5 , $G \lesssim_{s} \Gamma$ for every countable graph $G$. In particular $\Gamma^{*} \lesssim \Gamma$, thus $\Gamma^{*} \sim \Gamma$ and we have $\Gamma$ is indivisible thus, by Proposition $2.3 . \Gamma^{*}$ is indivisible.

To show $\Gamma^{*}$ is not symmetrically indivisible, let $c: \Gamma^{*} \rightarrow\{$ red, blue $\}$ be a colouring of $\Gamma^{*}$ defined by

$$
c(x)= \begin{cases}\text { red } & \text { if } x \in \Gamma \\ \text { blue } & \text { if } x \notin \Gamma\end{cases}
$$

Since there is no blue vertex of infinite degree there is no blue copy of $\Gamma$, and therefore also $\Gamma^{*}$ does not embed in the blue sub-graph. Let $\Gamma^{* \prime}$ be a red substructure isomorphic to $\Gamma^{*}$. By Lemmas 3.3 and 3.4, $\Gamma^{* \prime}$ has an automorphism that cannot be extended to an automorphism of $\Gamma^{*}$, and so $\Gamma^{* \prime}$ is not symmetrically embedded in $\Gamma^{*}$.

\subsection{Enumeration endowments.}

Throughout this subsection fix an ultrahomogeneous structure $U$ in a relational language $\mathcal{L}$ satisfying the pigeonhole property and a structure $U^{*}$ not symmetrically indivisible such that $U \lesssim U^{*} \lesssim U$. (For example, the random graph $\Gamma$ and $\Gamma^{*}$ defined above.)

Before we continue our construction, we give a general claim about the pigeonhole property:

Lemma 3.6. If $a_{1}, \ldots, a_{n}, b \in U$ are distinct and $g:\left\{a_{1}, \ldots, a_{n}\right\} \rightarrow U$ is a partial isomorphism, then the substructure whose universe is

$$
S:=\{x \in U \mid g \cup\langle b, x\rangle \text { is a partial isomorphism }\}
$$

is isomorphic to $U$, in particular, $S$ is infinite.

Proof. By ultrahomogeneity, $S$ is non-empty and since $a_{1}, \ldots, a_{n}, b$ are distinct, $g\left(a_{1}\right), \ldots, g\left(a_{n}\right) \notin S$. Let $s \in S$ and let $\widehat{g}=g \cup\{\langle b, s\rangle\}$. By the pigeonhole property, either $S$ or $U \backslash S$ is isomorphic to $U$. Assume towards a contradiction that there is an isomorphism $\phi: U \rightarrow U \backslash S$. Then

$$
\phi^{-1}\left\lceil\left\{\phi \circ g\left(a_{1}\right), \ldots, \phi \circ g\left(a_{n}\right)\right\}\right.
$$

is a partial isomorphism of $U \backslash S$, and thus by ultrahomogeneity, there is a $y \in U \backslash S$ such that

$$
f:=\left(\phi^{-1}\left\lceil\left\{\phi \circ g\left(a_{1}\right), \ldots, \phi \circ g\left(a_{n}\right)\right\}\right) \cup\{\langle\phi(s), y\rangle\}\right.
$$

is a partial isomorphism. Now $f \circ \phi \circ \widehat{g}$ is a partial isomorphism extending $g$ and $f \circ \phi \circ \widehat{g}(b) \in U \backslash S$, contradicting the definition of $S$.

Definition 3.7. For an $\mathcal{L}$ structure $\mathcal{M}$, we say an expansion of $\mathcal{M}$ to $\mathcal{L} \cup\{<\}$ is an enumeration endowment if $<$ is of order type $\omega$.

Note that two enumeration endowments of the same structure, even in the ultrahomogeneous context, are not necessarily isomorphic.

Definition 3.8. Recall that for a first-order relational structure $\mathcal{M}$, age $(\mathcal{M})$ is the class of all finite structures which are embeddable in $\mathcal{M}$.

Lemma 3.9. Let $A$ be a countable structure with age $(A) \subseteq \operatorname{age}(U)$. If $U^{<}, A^{<}$ are enumeration endowments of $U, A$ respectively, then $A^{<}$embeds into $U^{<}$(as $\mathcal{L} \cup\{<\}$-structures $)$.

In particular, $U^{<}$is indivisible. 
Proof. Let $\left\langle u_{i}: i \in \omega\right\rangle,\left\langle a_{i}: i \in \omega\right\rangle$ be enumerations of $U$ and $A$ respectively, compatible with the given enumeration endowments.

We construct the embedding inductively:

- Since age $(A) \subseteq \operatorname{age}(U)$, there is a $u \in U$ such that $\left\langle a_{0}, u\right\rangle$ is a partial isomorphism. Let $e_{0}:=\left\langle a_{0}, u\right\rangle$ for such a $u$.

- By Lemma 3.6, for every $i \in \omega$,

$$
S:=\left\{x \in U \mid e_{i} \cup\left\langle a_{i+1}, x\right\rangle \text { is a partial } \mathcal{L} \text {-isomorphism }\right\}
$$

is infinite. Choose $u \in S$ such that $e\left(a_{i}\right)<u$ in the enumeration endowment and let $e_{i+1}=e_{i} \cup\left\langle a_{i+1}, u\right\rangle$.

Let

$$
e:=\bigcup_{i \in \omega} e_{i}
$$

By the construction $e$ is an ascending union of $\mathcal{L}$-partial isomorphisms, so it is an $\mathcal{L}$-embedding. Furthermore it is order preserving - thus it is an $\mathcal{L} \cup\{<\}$-embedding.

Now to show $U^{<}$is indivisible, let $c: U \rightarrow\{$ red, blue $\}$. By indivisibility of $U$ as an $\mathcal{L}$-structure, there is a monochromatic $U^{\prime} \mathcal{L}$-isomorphic to $U$. Let $\left(U^{\prime}\right)^{<}$be the induced $\mathcal{L} \cup\{<\}$-structure on $U^{\prime}$. So $\left(U^{\prime}\right)^{<}$is an enumeration endowment of $U^{\prime}$ (not necessarily isomorphic to $U^{<}$). By the present lemma, $U^{<}$embeds into $\left(U^{\prime}\right)^{<}$ and it is monochromatic.

Remark 3.10. Note that since $\langle\omega,<\rangle$ is rigid, every enumeration endowment is rigid as well, thus in the context of enumerated graphs, symmetric indivisibility and indivisibility coincide.

Theorem 3.11. A reduct of a symmetrically indivisible structure to a sub-language is not necessarily symmetrically indivisible.

Proof. Consider $U$ and $U^{*}$ above. Assume for simplicity $U \subseteq U^{*}$. Let $\left(U^{*}\right)^{<}$be an enumeration endowment of $U^{*}$ and let $U^{<}$be the induced $\mathcal{L} \cup\{<\}$-substructure on $U$. Notice that $U^{<}$is an enumeration endowment of $U$ and thus by Lemma 3.9.

$$
\left(U^{*}\right)^{<} \lesssim U^{<}
$$

so by rigidity,

$$
\left(U^{*}\right)^{<} \lesssim_{s} U^{<} \lesssim_{s}\left(U^{*}\right)^{<}
$$

By Lemma 3.9 and by rigidity $U^{<}$is symmetrically indivisible and thus by Proposition 2.3. so is $\left(U^{*}\right)^{<}$. But $U^{<} \uparrow \mathcal{L}=U^{*}$ is not symmetrically indivisible.

Acknowledgement. The work in this paper is part of the author's M.Sc. thesis, prepared under the supervision of Assaf Hasson. The author would like to gratefully acknowledge him for presenting the question discussed in the paper, as well as the great help and support along the way. Thanks are also due to Menachem Kojman for his help in a preliminary version of the paper. The author was partially supported by an Israel Science Foundation grant number 1156/10. 


\section{REFERENCES}

[Cam10] Peter J. Cameron. The pigeonhole property. Available at http://cameroncounts.wordpress.com/2010/10/11/the-pigeonhole-property/, 2010.

[EZS91] Mohamed M. El-Zahar and Norbert W. Sauer. Ramsey-type properties of relational structures. Discrete Math., 94(1):1-10, 1991.

[EZS93] Mohamed M. El-Zahar and Norbert W. Sauer. On the divisibility of homogeneous directed graphs. Canad. J. Math., 45(2):284-294, 1993.

[Fra00] Roland Fraïssé. Theory of relations, volume 145 of Studies in Logic and the Foundations of Mathematics. North-Holland Publishing Co., Amsterdam, revised edition, 2000. With an appendix by Norbert Sauer.

[GK11] Stefan Geschke and Menachem Kojman. Symmetrized induced Ramsey theory. Graphs Combin., 27(6):851-864, 2011.

[Hen71] C. Ward Henson. A family of countable homogeneous graphs. Pacific J. Math., 38:69-83, 1971.

[HKO11] Assaf Hasson, Menachem Kojman, and Alf Onshuus. On symmetric indivisibility of countable structures. Contemp. Math., 558(1):453-466, 2011.

[KR86] Péter Komjáth and Vojtěch Rödl. Coloring of universal graphs. Graphs Combin., 2(1):55-60, 1986.

[Rad64] Richard Rado. Universal graphs and universal functions. Acta Arith., 9:331-340, 1964.

[Sau14] N. Sauer. Age and weak indivisibility. European Journal of Combinatorics, 37(0):24 31, 2014. Relational structures, ordered sets and graphs.

E-mail address: mein@math.bgu.ac.il

Department of Mathematics, Ben-Gurion University of the Negev, P.O.B. 653, Be’er SHEVA 8410501, ISRAEL 